\title{
Ueber die Anziehung einer mit Masse belegten ab- wickelbaren Fläche auf einen materiellen Punkt.
}

(Von Herrn Mehler zu Fraustadt.)

Wenn ein materieller Punkt von jedem Elemente einer krummen Oberfläche proportional einer Potenz der Entfernung und proportional der auf dem Elemente vertheilten Masse angezogen wird, so lassen sich die Componenten der Gesammtanziehung, welche er erfährt, bekanntlich als die partiellen Differentialquotienten ein und desselben Doppelintegrales darstellen. In diesem Doppelintegrale ist keine der beiden Integrationen ausführbar, so lange über die Natur der Fläche und die Art der Massenvertheilung nicht besondere, einfache Vorausselzungen gelten. Der Zweck der folgenden Zeilen ist, auf einen Fall aufmerksam zu machen, in welchem die Aufgabe für eine beliebige abwickelbare Fläche sich auf Quadraturen zurückführen läfst, und die einfachen Resultate herzuleiten, welche daraus für Kegelflächen und insbesondere für den geraden Kegel sich ergeben.

Die rechtwinkligen Coordinaten $\xi, \eta, \zeta$ eines Punktes der Wendungscurve der abwickelbaren Fläche seien als Functionen einer Veränderlichen $\alpha$ gegeben vermöge der Gleichungen:

$$
\text { (1.) } \xi=\varphi \alpha, \quad \eta=\psi \alpha, \quad \zeta=\chi \alpha \text {, }
$$

und unter $\alpha$ möge der Einfachheit wegen der Bogen der Curve, von einem festen Punkte aus gerechnet, verstanden werden. Alsdann sind $\varphi^{\prime} \alpha, \psi^{\prime} \alpha, \chi^{\prime} \alpha$ die Cosinus der Winkel, welche die Tangente im Punkte $(\xi, \eta, \zeta)$, nach der Seite hin gezogen, nach welcher der Bogen wächst, mit den Coordinatenaxen bildet, und man hat die Bedingungsgleichung:

$$
\left(\varphi^{\prime} \alpha\right)^{2}+\left(\psi^{\prime} \alpha\right)^{2}+\left(\chi^{\prime} \alpha\right)^{2}=1,
$$

und daher auch die folgende:

$$
\text { (2'.) } \quad \varphi^{\prime} \alpha \varphi^{\prime \prime} \alpha+\psi^{\prime} \alpha \psi^{\prime \prime} \alpha+\chi^{\prime} \alpha \chi^{\prime \prime} \alpha=0 \text {. }
$$

Trägt man auf irgend einer Tangente vom Berührungspunkte aus eine Strecke $u$ ab, so werden die Coordinaten $x, y, z$ des Endpunktes, der stets ein Punkt der abwickelbaren Fläche ist, als Functionen von $\alpha$ und $u$ bestimmt durch die Gleichungen:

(3.) $x=\varphi \alpha+u \varphi^{\prime} \alpha, \quad y=\psi \alpha+u \psi^{\prime} \alpha, \quad z=\chi \alpha+u \chi^{\prime} \alpha$. 
Läfst man hierin $\alpha$ den ganzen Bogen der Wendungscurve durchlaufen und $u$ alle möglichen positiven Werthe annehmen, so erhält man nur die Punkte auf dem einen von den beiden Theilen, in welche die abwickelbare Fläche durch die Wendungscurve getheilt wird. Will man auch den anderen Theil der Fläche erschöpfen, so mufs man die Strecke $u$ auch auf den Richtungen der Tangenten abtragen, welche mit den Axen Winkel bilden, deren Cosinus respective $=-\varphi^{\prime} \alpha,-\psi^{\prime} \alpha,-\chi^{\prime} \alpha$ sind; oder man mufs in (3.) der Gröfse $\boldsymbol{u}$ auch alle $W$ erthe von 0 bis $-\infty$ ertheilen.

Die abwickelbare Fläche sei so mit Masse belegt, dafs die Dichtigkeit $\boldsymbol{k}$ für verschiedene Punkte auf derselben Tangente der Entfernung vom Berührungspunkte umgekehrt proportional ist, während sie von Tangente zu Tangente sich noch stetig ändern kann. Dieses Geselz, das die Dichligkeit befolgen soll, kann dargestellt werden durch die Formel:

$$
\text { (4.) } k=\frac{f \alpha}{ \pm u}
$$

worin $f \alpha$ eine beliebige Function von $\alpha$ und $\pm u$ den absoluten Werth der Entfernung bezeichnet. Die Kraft, mit welcher die Masseneinheit einen materiellen Punkt in der Entfernung $r$ anzieht, sei $=\frac{1}{r^{p}}$. Es soll die Anziehung eines Theiles der Fläche berechnet werden, der zwischen zwei, zu den Bogen $\alpha_{0}$ und $\alpha_{1}$ gehörigen Tangenten enthalten ist.

Es seien $\boldsymbol{a}, \boldsymbol{b}, \boldsymbol{c}$ die Coordinaten des angezogenen Punktes, $\boldsymbol{A}, \boldsymbol{B}, \boldsymbol{C}$ die Componenten der Anziehung, $d \omega$ das Oberflächenelement für den Punkt $(x, y, z), r$ die Entfernung der Punkte $(a, b, c)$ und $(x, y, z)$, so dafs:

$$
\text { (5.) } \quad r^{2}=(a-x)^{2}+(b-y)^{2}+(c-z)^{2} \text {; }
$$

dann hat man für $\boldsymbol{A}$ den Ausdruck:

$$
\text { (6.) } \quad A=-\int \frac{1}{r^{p}} \frac{a-x}{r} k d \omega
$$

und ganz ähnliche für $B$ und $C$. Setzt man:

$$
\text { (7.) } T=\frac{1}{p-1} \int \frac{k d \omega}{r^{p-1}}
$$

so wird:

$$
\text { (8.) } \quad \boldsymbol{A}=\frac{\partial \boldsymbol{T}}{\partial a}, \quad \boldsymbol{B}=\frac{\partial \boldsymbol{T}}{\partial b}, \quad \boldsymbol{C}=\frac{\partial \boldsymbol{T}}{\partial c},
$$

und die Aufgabe besteht jetzt in der Bestimmung des Integrales $\boldsymbol{T}$. Für das Oberflächenelement ergiebt sich durch geometrische Betrachtungen oder mit 
242 Mehler, über eine Aufgabe der Anziehung für abwickelbare Flächen.

Hülfe der bekannten Formel:

$$
d \omega=
$$

$d \alpha d u \sqrt{\left[\left(\frac{\partial x}{\partial \alpha}\right)^{2}+\left(\frac{\partial y}{\partial \alpha}\right)^{2}+\left(\frac{\partial z}{\partial \alpha}\right)^{2}\right]\left[\left(\frac{\partial x}{\partial u}\right)^{z}+\left(\frac{\partial y}{\partial u}\right)^{2}+\left(\frac{\partial z}{\partial u}\right)^{2}\right]-\left[\frac{\partial x}{\partial \alpha} \frac{\partial x}{\partial u}+\frac{\partial y}{\partial \alpha} \frac{\partial y}{\partial u}+\frac{\partial z}{\partial \alpha} \frac{\partial z}{\partial u}\right]^{2}}$ sehr leicht der Ausdruck:

$$
d \omega= \pm u \sqrt{\left(\varphi^{\prime \prime} \alpha\right)^{2}+\left(\psi^{\prime \prime} \alpha\right)^{2}+\left(\chi^{\prime \prime} \alpha\right)^{2}} d \alpha d u,
$$

welcher durch Einführung des Krümmungshalbmessers $\varrho$ der Wendungscurve die Form annimmt:

Daher wird:

$$
d \omega=\frac{ \pm u d \alpha d u}{\varrho}
$$

und folglich:

$$
\text { (9.) } \quad \dot{k} d \omega=\frac{f \alpha d \alpha}{\varrho} d u \text {, }
$$

Aus (5.) und (3.) folgt:

$$
\text { (10.) } T=\frac{1}{p-1} \int_{\alpha_{0}}^{\alpha_{1}} \frac{f \alpha d a}{\varrho} \int_{-\infty}^{\infty} \frac{d u}{r^{p-1}} \text {. }
$$

$$
\boldsymbol{r}^{2}=\left(\boldsymbol{a}-\varphi \alpha-u \varphi^{\prime} \alpha\right)^{2}+\left(b-\psi \alpha-u \psi^{\prime} \alpha\right)^{2}+\left(c-\chi \alpha-u \chi^{\prime} \alpha\right)^{2} .
$$

Setzt man daher:

$$
\text { (11.) }\left\{\begin{array}{l}
(a-\varphi \alpha)^{2}+(b-\psi \alpha)^{2}+(c-\chi \alpha)^{2}=l^{2} \\
(a-\varphi \alpha) \varphi^{\prime} \alpha+(b-\psi \alpha) \psi^{\prime} \alpha+(c-\chi \alpha) \chi^{\prime} \alpha=m,
\end{array}\right.
$$

so dafs $l$ den Leitstrahl von einem Punkte $(\varphi \alpha, \psi \alpha, \chi \alpha)$ der Wendungscurve nach $(a, b, c)$, und $m$ die Projection desselben auf die zugehörige Tangente bedeutet, so wird:

$$
r^{2}=l^{2}-2 m u+u^{2}=l^{2}-m^{2}+(u-m)^{2},
$$

also, wenn $\int_{-\infty}^{\infty} \frac{d u}{r^{p-1}}$ durch $U$ bezeichnet wird :

Durch die Substitution:

$$
U=\int_{-\infty}^{\infty} \frac{d u}{\left[l^{2}-m^{2}+(u-m)^{2}\right]^{\frac{p-1}{2}}} .
$$

folgt hieraus :

$$
u=\boldsymbol{m}+\left(\boldsymbol{l}^{2}-\boldsymbol{m}^{2}\right)^{\frac{1}{2}} v
$$

$$
U=\left(l^{2}-m^{2}\right)^{1-\frac{p}{2}} \int_{-\infty}^{\infty} \frac{d v}{\left(1+v^{2}\right)^{\frac{p-1}{2}}}=2\left(l^{2}-m^{2}\right)^{1-\frac{p}{2}} \int_{0}^{\infty} \frac{d v}{\left(1+v^{2}\right)^{\frac{p-1}{2}}} .
$$

Man sieht jetzt beiläufig, dafs $\mu>2$ sein mufs, damit $U$ und folglich $\boldsymbol{T}$ nicht 
Mehler, über eine Aufgabe der Anziehung für abwickelbare Flächen. 243 unendlich werde. Setzt man $v=w^{\ddagger}$, so erhält man:

$$
\begin{aligned}
2 \int_{0}^{\infty} \frac{d v}{\left(1+v^{2}\right)^{\frac{p-1}{2}}} & =\int_{0}^{\infty} \frac{w^{\frac{1}{2}-1} d w}{(1+w)^{\frac{1}{2}+\left(\frac{p}{2}-1\right)}}=\left(\frac{1}{2}, \frac{p}{2}-1\right) \\
& =\frac{\Gamma\left(\frac{1}{2}\right) \Gamma\left(\frac{p}{2}-1\right)}{\Gamma\left(\frac{p-1}{2}\right)}=\frac{\sqrt{ } \pi \Gamma\left(\frac{p}{2}-1\right)}{\Gamma\left(\frac{p-1}{2}\right)}
\end{aligned}
$$

also ist:

$$
U \operatorname{oder} \int_{-\infty}^{\infty} \frac{d u}{r^{p-1}}=\frac{\sqrt{ } \pi \Gamma\left(\frac{p}{2}-1\right)}{\Gamma\left(\frac{p-1}{2}\right)}\left(l^{2}-m^{2}\right)^{1-\frac{p}{2}} .
$$

Durch Einführung dieses Werthes in (10.) erhält man, unter Berücksichtigung, dafs :

für $\boldsymbol{T}$ den Ausdruck:

$$
\left(\frac{p-1}{2}\right) \Gamma\left(\frac{p-1}{2}\right)=\Gamma\left(\frac{p+1}{2}\right)
$$

$$
\text { (12.) } \quad \boldsymbol{T}=\frac{\sqrt{ } \pi \Gamma\left(\frac{p}{2}-1\right)}{2 \Gamma\left(\frac{p+1}{2}\right)} \int_{\alpha_{0}}^{\alpha_{1}} \frac{d \alpha f \alpha}{\varrho}\left(l^{2}-m^{2}\right)^{1-\frac{p}{2}},
$$

und wenn man diese Gleichung partiell nach $a, b, c$ differentiirt und bemerkt, dafs nach (11.):

$$
\frac{\partial\left(l^{2}\right)}{\partial u}=2(a-\varphi \alpha), \quad \frac{\partial\left(m^{2}\right)}{\partial a}=2 m \varphi^{\prime} \alpha, \text { u. s. w., }
$$

so ergeben sich für die Attractionscomponenten die Formeln:

$$
\text { (13.) }\left\{\begin{array}{l}
A=-\frac{\sqrt{ } \pi \Gamma\left(\frac{p}{2}\right)}{\Gamma\left(\frac{p+1}{2}\right)} \int_{\alpha_{0}}^{\alpha_{1}} \frac{d \alpha f \alpha}{\rho} \cdot \frac{a-\varphi \alpha-m \varphi^{\prime} \alpha}{\left(l^{2}-m^{2}\right)^{\frac{p}{2}}} \\
B=-\frac{\sqrt{ } \pi \Gamma\left(\frac{p}{2}\right)}{\Gamma\left(\frac{p+1}{2}\right)} \int_{\alpha_{0}}^{\alpha_{1}} \frac{d \alpha f \alpha}{\varrho} \cdot \frac{b-\psi \alpha-m \psi^{\prime} \alpha}{\left(l^{2}-m^{2}\right)^{\frac{p}{2}}} \\
C=-\frac{\sqrt{ } \pi \Gamma\left(\frac{p}{2}\right)}{\Gamma\left(\frac{p+1}{2}\right)} \int_{\alpha}^{\alpha_{1}} \frac{d \alpha f \alpha}{\rho} \cdot \frac{c-\chi \alpha-m \chi^{\prime} \alpha}{\left(l^{2}-m^{2}\right)^{\frac{p}{2}}}
\end{array}\right.
$$

Die Grófsen unter den Integralzeichen haben eine einfache Bedeutung, welche eine geometrische Interpretation der gewonnenen Formeln gestattet. 
244 Mehler, über eine Aufgabe der Anziehung für abwickelbare Flächen.

Es ist nämlich $\left(l^{2}-m^{2}\right)^{\frac{1}{2}}$ die Länge des Lothes, das man von $(a, b, c)$ auf die Tangente der Wendungscurve im Punkte $(\varphi \alpha, \psi \alpha, \chi \alpha)$ fällt; es sind ferner $\varphi \alpha+m \varphi^{\prime} \alpha, \psi \alpha+m \psi^{\prime} \alpha, \chi \alpha+m \chi^{\prime} \alpha$ die Coordinaten des Fufspunktes dieses Lothes, und folglich $a-\varphi \alpha-m \varphi^{\prime} \alpha, b-\psi \alpha-m \psi^{\prime} \alpha, c-\chi \alpha-m \chi^{\prime} \alpha$ die Projectionen desselben auf die Coordinatenaxen. Bezeichnet man daher die Länge des Lothes mit $n$ und durch $\lambda, \mu, \nu$ die Winkel, welche es mit den Axen bildet, und schreibt $\boldsymbol{P}$ statt des numerischen Factors $\frac{\sqrt{ } \pi \Gamma\left(\frac{p}{2}\right)}{\Gamma\left(\frac{p+1}{2}\right)}$, so wird:

$$
\boldsymbol{A}=\int_{\alpha_{0}}^{\alpha_{1}} \frac{\boldsymbol{P} d \alpha f \alpha}{\varrho} \cdot \frac{1}{n^{p-1}} \cdot \cos \lambda
$$

u. s. w.

Also kann die Anziehung der abwickelbaren Fläche ersetzt werden durch die Anziehung der Fufspunktencurve ihrer Wendungscurve für den angezogenen Punkt als Pol, vorausgesetzt dafs jedes $d \alpha$ entsprechende Bogenelement der Fufspunktencurve die Masse $\frac{P d \alpha f \alpha}{\rho}$ erhält und den materiellen Punkt umgekehrt proportional, nicht der $p^{\text {ten }}$, sondern der $(p-1)^{\text {ten }}$ Potenz der Entfernung anzieht.

Auch die Art der Massenvertheilung auf der anziehenden Curve läfst eine einfache geometrische Deutung zu. Trägt man nämlich auf den Tangenten der Wendungscurve von den Berührungspunkten aus Stücke von der constanten Länge $\boldsymbol{P}$ ab, so dafs die Endpunkte eine neue Curve auf der abwickelbaren Fläche bestimmen, so enthält der Streifen der Oberfläche, welcher von zwei unendlich nahen Tangenten und den zugehörigen Bogenelementen der Wendungs - und der eben construirten Curve begrenzt wird, stets gerade diejenige Masse, welche dem zwischen den beiden nämlichen Tangenten gelegenen Bogenelemente der Fufspunktencurve mitzutheilen ist. Denn vermöge (9.) ist die Masse jenes Streifens

$$
=\frac{d \alpha f \alpha}{\rho} \int_{0}^{P} d u=\frac{P d \alpha f \alpha}{\varrho} .
$$

Ist $p \leqq 2$, so haben die angestellten Rechnungen keine Gültigkeit mehr. Behandelt man aber in diesem Falle unmittelbar die Attractionscomponenten auf dieselbe Weise, wie dies mit $\boldsymbol{T}$ geschah, so findet man ohne Mühe, dafs sie noch durch die Formeln (13.) ausgedrückt werden; wenn $p$ zwischen 2 
Mehler, über eine Aufgabe der Anziehung für abwickelbare Flächen. 245

und 1 liegt, dafs sie dagegen unbestimmt oder unendlich grofs werden, wenn $p \leqq 1$ ist.

Für $p=2, d$. h. für den Fall des Newtonschen Attractionsgesetzes, lehrt die Gleichung (12.), dafs das Potential der abwickelbaren Fläche unendlich grofs ist; für die Componente $\boldsymbol{A}$ aber ergiebt sich aus (13.) der endliche Werth:

$$
A=-2 \int_{\alpha_{0}}^{\alpha_{1}} \frac{d \alpha f \alpha}{\varrho} \cdot \frac{a-\varphi \alpha-m \varphi^{\prime} \alpha}{l^{2}-m^{2}}
$$

und ähnliche für $\boldsymbol{B}$ und $\boldsymbol{C}$. Man sieht, dafs die Attractionscomponenten hier die nach $\boldsymbol{a}, \boldsymbol{b}, \boldsymbol{c}$ genommenen partiellen Differentialquotienten des Integrales

$$
T^{\prime}=-\int_{\alpha_{0}}^{\alpha_{1}} \frac{d \alpha f \alpha}{\rho} \log \left(l^{2}-m^{2}\right)
$$

sind, und man überzeugt sich leicht, dafs $\boldsymbol{T}^{\prime}$ aus $\boldsymbol{T}$ hervorgeht, wenn man von $\boldsymbol{T}$ die Constante

$$
\frac{\sqrt{ } \pi \Gamma\left(\frac{p}{2}-1\right)}{2 \Gamma\left(\frac{p+1}{2}\right)} \int_{\alpha_{0}}^{\alpha_{1}} \frac{d \alpha f \alpha}{\varrho}
$$

subtrahirt und alsdann erst $p=2$ setzt.

Um die für jede abwickelbare Fläche gültigen Formeln (13.) auf Kegelflächen, für welche die Wendungscurve sich auf einen Punkt reducirt, anwenden zu können, setze man zunächst:

$$
\varphi \alpha=\varepsilon \Phi \frac{\alpha}{\varepsilon}, \quad \psi \alpha=\varepsilon \Psi \frac{\alpha}{\varepsilon}, \quad \chi \alpha=\varepsilon X \frac{\alpha}{\varepsilon},
$$

also:

$$
\varphi^{\prime} \alpha=\Phi^{\prime} \frac{\alpha}{\varepsilon}, \quad \psi^{\prime} \alpha=\Psi^{\prime} \frac{\alpha}{\varepsilon}, \quad \chi^{\prime} \alpha=X^{\prime} \frac{\alpha}{\varepsilon},
$$

und darauf :

$$
\frac{\alpha}{\varepsilon}=\beta, \quad \varepsilon=0 .
$$

Alsdann gehen die Gleichungen (3.) über in:

$$
\text { (14.) } \quad x=u \Phi^{\prime} \beta, \quad y=u \Psi^{\prime} \beta, \quad z=u X^{\prime} \beta
$$

und stellen eine beliebige Kegelfläche dar, deren Mittelpunkt im Anfangspunkte liegt; es wird:

$$
\begin{aligned}
\frac{d \alpha}{\rho} & =d \beta \sqrt{\left(\Phi^{\prime \prime} \beta\right)^{2}+\left(\Psi^{\prime \prime} \beta\right)^{2}+\left(X^{\prime \prime} \beta\right)^{2}}=\frac{d \beta}{P}, \quad f a=F \beta, \\
l^{2} & =a^{2}+b^{2}+c^{2}, \quad m=a \Phi^{\prime} \beta+b \Psi^{\prime} \beta+c X^{\prime} \beta,
\end{aligned}
$$

Journal für Mathematik Bd. LVIII. Heft 3. 
246 Mehler, über eine Aufgabe der Anziehung für abwickelbare Flächen.

und die Gleichungen (13.) geben für die Componenten der Anziehung, welche ein zwischen zwei beliebigen Seitenlinien enthaltenes Stück der Kegelfläche ausübt :

$$
\text { (17.) } \quad \boldsymbol{A}=-\frac{\sqrt{ } \pi \Gamma\left(\frac{p}{2}\right)}{\Gamma\left(\frac{p+1}{2}\right)} \int_{\beta_{0}}^{\cdot \beta_{1}} \frac{d \beta F \beta}{P} \frac{a-m \Phi^{\prime} \beta}{\left(l^{2}-m^{2}\right)^{\frac{p}{2}}} \text {. }
$$

u. s. w.

Die Gröfse $l$, welche jetzt die Entfernung des angezogenen Punktes vom Anfangspunkte bedeutel, ist von $\beta$ unabhängig, und es sind $l$ und $m$ homogene Functionen des ersten Grades von $a, b$ und $c$. Setzt man demnach:

$$
a=l \cos \lambda, \quad b=l \sin \lambda \cos \mu, \quad c=l \sin \lambda \sin \mu,
$$

so nehmen die Ausdrücke für die Attractionscomponenten die Form an:

$$
\boldsymbol{A}=\frac{\boldsymbol{L}}{l^{p-1}}, \quad \boldsymbol{B}=\frac{\boldsymbol{M}}{l^{p-1}}, \quad \boldsymbol{C}=\frac{\boldsymbol{N}}{l^{p-1}},
$$

worin $\boldsymbol{L}, \boldsymbol{M}, \boldsymbol{N}$ nur von $\lambda$ und $\mu$ abhängen, also für Punkte auf derselben durch den Ursprung gehenden Geraden constant sind. Daher gilt der Satz:

Die Anziehung der Kegelfläche auf verschiedene Punkte desselben vom Ursprung ausgehenden Radiusvectors ist für alle gleich gerichtet und der $(p-1)^{\text {ten }}$ Potenz des Abstandes vom Mittelpunkte umgekehrt proportional.

Gilt das Newtonsche Anziehungsgesetz, so erhält die nach dem Mittelpunkt gerichtete Componente $\boldsymbol{Q}$ vermöge (17.) den Werth:

$$
\text { (18.) } \quad \boldsymbol{Q}=-\boldsymbol{A} \frac{a}{l}-\boldsymbol{B} \frac{b}{l}-\boldsymbol{C} \frac{c}{l}=\frac{2}{l} \int_{\beta_{0}}^{\beta_{1}} \frac{d \beta F \beta}{P} \text {. }
$$

Diese Componente ist also constant für alle gleich weit vom Mittelpunkt entfernte Punkte, und für Punkte, die ungleichen Abstand haben, diesem Abstande umgekehrt proportional.

Wir betrachten noch besonders den sehr einfachen Fall, dafs der Kegel ein Umdrehungskegel, dafs die Dichtigkeit für alle Seitenlinien in gleichem Abstand vom Mittelpunkte auch gleich grofs, und zwar im Abstand 1 gleich 1 ist, und dafs die Anziehung nach dem Newtonschen Gesetze erfolgt.

Die $\boldsymbol{X}$-Axe sei die Rotationsaxe des Kegels, $\varepsilon$ der constante Winkel derselben mit den Seitenlinien und $\beta$ der Winkel, den die Projection einer Seitenlinie auf die Ebene der $\boldsymbol{Y} \boldsymbol{Z}$ mit $\operatorname{der}$ Axe der $\boldsymbol{Y}$ bildet. Dann wird:

$$
\begin{array}{cl}
\Phi^{\prime} \beta=\cos \varepsilon, & \Psi^{\prime} \beta=\sin \varepsilon \cos \beta, \quad X^{\prime} \beta=\sin \varepsilon \sin \beta, \\
\frac{1}{P}=\sin \varepsilon, & F=1, \quad \beta_{0}=0, \quad \beta_{1}=2 \pi, \quad p=2,
\end{array}
$$


Mehler, über eine Aufgabe der Anziehung für abwickelbare Flächen. 247

und für $m$ erhält man aus (16.), wenn:

und:

(19.) $\quad a=l \cos \lambda, \quad b=l \sin \lambda \cos \mu, \quad c=l \sin \lambda \sin \mu$

(20.) $\quad \cos \lambda \cos \varepsilon+\sin \lambda \sin \varepsilon \cos (\beta-\mu)=\cos \vartheta$

gesetzt wird:

$$
\text { (21.) } \quad m=l \cos \vartheta
$$

Durch Einführung dieser Werthe ergiebt sich aus (17.):

$$
A=-\frac{2 \sin \varepsilon}{l} \int_{0}^{2 \pi} \frac{\cos \lambda-\cos \varepsilon \cos \vartheta}{1-\cos ^{2} \vartheta} d \beta
$$

Dieser Ausdruck läfst sich darstellen in der Form:

$$
\boldsymbol{A}=-\frac{\sin \varepsilon}{l}\left\{\int_{0}^{2 \pi} \frac{\cos \varepsilon+\cos \lambda}{1+\cos \vartheta} d \beta-\int_{0}^{2 \pi} \frac{\cos \varepsilon-\cos \lambda}{1-\cos \vartheta} d \beta\right\} \text {. }
$$

Substituirt man hierin für $\cos \vartheta$ seinen Werth aus (20.) und setzt $\beta-\mu=2 \gamma$, so erhält man nach einigen Umformungen:

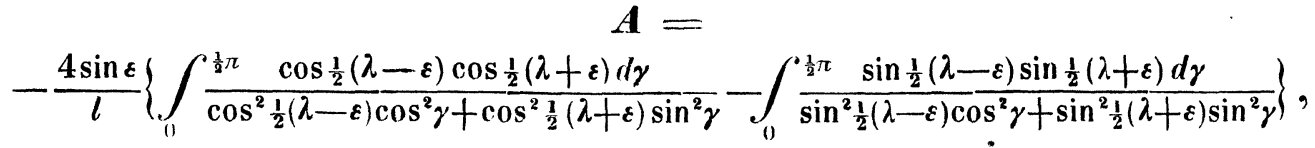

also ist:

$$
A=-\frac{4 \sin \varepsilon}{l}\left\{\operatorname{arctg}\left(\frac{\cos \frac{1}{2}(\lambda+\varepsilon)}{\cos \frac{1}{2}(\lambda-\varepsilon)} \operatorname{tg} \frac{1}{2} \pi\right)-\operatorname{arctg}\left(\frac{\sin \frac{1}{2}(\lambda+\varepsilon)}{\sin \frac{1}{2}(\lambda-\varepsilon)} \operatorname{tg} \frac{1}{2} \pi\right)\right\} .
$$

Jeder der beiden Kreisbogen, deren Differenz in der Parenthese steht, erhält den Werth $+\frac{1}{2} \pi$ oder $-\frac{1}{2} \pi$, je nachdem sein, in jedem Falle unendlich grofses Argument positiv oder negativ ist. Nun kann man alle möglichen Lagen des angezogenen Punktes erschöpfen, wenn man $\mu$ von 0 bis $2 \pi, \lambda$ von 0 bis $\frac{1}{2} \pi$ und $l$ von $-\infty$ bis $\infty$ variiren läfst, und zwar wird der Punkt aufserhalb oder innerhalb des Kegels liegen, je nachdem man $\lambda$ zwischen $\varepsilon$ und $\frac{1}{2} \pi$ oder zwischen 0 und $\varepsilon$ wählt. Demnach ist statt des ersten Kreisbogens offenbar stets $\frac{1}{2} \pi$, statt des zweiten dagegen $\frac{1}{2} \pi$ für einen äufseren und $-\frac{1}{2} \pi$ für einen inneren Punkt zu setzen. Also hat man für einen äu/seren Punkt:

und für einen inneren Punkt:

$$
\text { (23.) } \boldsymbol{A}=0 \text {, }
$$

$$
\text { (23'.) } \quad A=-\frac{4 \pi \sin \varepsilon}{l} .
$$

Um auch die beiden anderen Componenten der Anziehung zu berechnen, benutzt man am einfachsten die Gleichungen:

$$
\left\{\begin{aligned}
\boldsymbol{A} a+B b+C \boldsymbol{c} & =-4 \pi \sin \varepsilon \\
\boldsymbol{B} \boldsymbol{c}-\boldsymbol{C b} & =0
\end{aligned}\right.
$$


248 Mehler, über eine Aufgabe der Anzichung für abwickelbare Flüchen.

von denen die erste aus (18.), die zweite aber daraus folgt, dafs die Richtung der Resultante die Axe des Kegels schneiden mufs. Mit Hülfe derselben erhält man für einen aufserhalb gelegenen Punkt:

(25.)

$$
\left\{\begin{array}{l}
\boldsymbol{A}=0 \\
\boldsymbol{B}=-4 \pi \sin \varepsilon \frac{b}{b^{2}+c^{2}}=-\frac{4 \pi \sin \varepsilon \cos \mu}{l \sin \lambda} \\
\boldsymbol{C}=-4 \pi \sin \varepsilon \frac{c}{b^{2}+c^{2}}=-\frac{4 \pi \sin \varepsilon \sin \mu}{l \sin \lambda}
\end{array}\right.
$$

Die Anziehung ist also senkrecht nach der Axe hin gerichtet, und ihre Intensität der Entfernung des angezogenen Punktes von der Axe umgekehrt proportional.

Für einen im Innern des Kegels befindlichen Punkt gelten dagegen die Formeln:

$$
\left(25^{\prime} .\right) .\left\{\begin{array}{l}
\boldsymbol{A}=-\frac{4 \pi \sin \varepsilon}{l}, \\
\boldsymbol{B}=-\frac{4 \pi \sin \varepsilon b}{l(l+a)}=-\frac{4 \pi \sin \varepsilon \operatorname{tg} \frac{1}{2} \lambda \cos \mu}{l}, \\
\boldsymbol{C}=-\frac{4 \pi \sin \varepsilon c}{l(l+a)}=-\frac{4 \pi \sin \varepsilon \operatorname{tg} \frac{1}{2} \lambda \sin \mu}{l} .
\end{array}\right.
$$

Die erste dieser Gleichungen zeigt, dafs die mit der Axe des Kegels parallele Componente nach der Seite hin gerichtet ist, auf welcher der Mittelpunkt des Kegels sich befindet. Die nach dem Mittelpunkt hin gerichtete Componente hat nach (18.) den Werth $\frac{4 \pi \sin \varepsilon}{l}$, ist also gleich grofs mit der parallel zur Axe gerichteten. Bemerkt man noch, dafs die Ebene dieser beiden Componenten auch die Resultante enthält, so gelangt man zu der folgenden einfachen Construction der Resultante für einen innern Punkt:

Man ziehe von dem angezogenen Punkte eine Gerade nach dem Miltelpunkte und eine andere parallel zur Axe, trage auf. ihnen gleiche Strecken ab von der Länge $\frac{4 \pi \sin \varepsilon}{l}$ und construire mit denselben einen Rhombus: alsdann wird die Resultante der Gröfse und Richtung nach dargestellt durch die Diagonale dieses Rhombus.

Die Richtung der Resultante halbirt folglich den spitzen Winkel, welchen der Radiusvector nach dem Mittelpunkte und die Parallele zur Axe mit einander bilden.

Fraustadt, im April 1860. 